\title{
MORPHOLOGY INFLUENCE ON WETTABILITY AND WETTING DYNAMICS OF ZnO NANOSTRUCTURE ARRAYS
}

\author{
V. Gerbreders ${ }^{1}$, M. Krasovska1 ${ }^{1 *}$, I. Mihailova ${ }^{1}$, E. Sledevskis ${ }^{1}$, A. Ogurcovs ${ }^{1,2}$, \\ E. Tamanis ${ }^{1}$, V. Auksmuksts' ${ }^{1}$ A. Bulanovs ${ }^{1}$, V. Mizers ${ }^{1}$
}

${ }^{1}$ G. Liberts' Innovative Microscopy Centre, Department of Technology, Institute of Life Sciences and Technology, Daugavpils University,

1 Parades Str., Daugavpils, LV-5401, LATVIA

${ }^{2}$ Thin Films Laboratory, Institute of Solid State Physics, University of Latvia, 8 Kengaraga Str., Riga, LV-1063, LATVIA

*e-mail: marina.krasovska@du.Iv

Changes in nanostructure morphology and size may result in very different surface wettability. In this research, the impact of different morphological parameters on the wetting dynamics of $\mathrm{ZnO}$ nanostructured layers is studied. Six different morphologies are chosen to determine the specific wetting processes of $\mathrm{ZnO}$ nanostructures: nanoneedles, small diameter rods, large diameter rods, nanotubes, nanoplates, and plain thin films. Wetting dynamics is investigated using conventional sessile drop technique and a novel approach based on electrochemical impedance spectroscopy. The results show that the surface of nanostructured $\mathrm{ZnO}$ thin films exhibits both hydrophilic and hydrophobic wetting behaviour, depending on nanostructure form, size, and orientation. $\mathrm{ZnO}$ nanostructure arrays are a promising platform for electrochemical and optical sensing in aqueous solutions. The full and effective use of the sensor working surface can be ensured only under the condition of complete wetting of the nanostructured layer. Therefore, it is important to take into account the peculiarities of the wetting process of a specific morphology of nanostructures.

Keywords: Electrochemical impedance spectroscopy, nanostructures, water contact angle, wettability, $\mathrm{ZnO}$. 
$\mathrm{ZnO}$ nanostructures have attracted considerable attention over the past few years because of noticeable properties coupled with efficient flexibility in morphology. These fascinating properties make them highly desirable for applications in chemisensors and biosensors, with good sensitivity and low detection limits for different analytes [1]-[4]. As it is well known, biosensors are instrumental analytical devices, which convert signals from molecular recognition elements into electrical or other types of signals, with the response being proportional to the target analyte concentration in the sample. $\mathrm{ZnO}$ nanostructures have large surface-to-volume ratios [5], [6] and, therefore, can deposit much larger amounts of bioanalytical materials on the nanostructures than on planar films, leading to an increase in signal intensity and sensitivity of the biosensor. Numerous methods for obtaining $\mathrm{ZnO}$ nanostructures, including gas phase [7] and solution phase methods [8], have led to the formation of structures with wurtzite lattices but different morphologies, which can be varied in diverse ways: by seed layers, acidity of growth solutions, various additives, or adjustment of other growth parameters [1], [9]. For sensor application, the optimal morphology is one that provides the maximum signal-to-noise ratio when examining the analyte under otherwise identical conditions. Therefore, achieving excellent sensitivity, selectivity, and reproducibility in analyte analysis is one of the main tasks in developing new and improved analytical methods.

Furthermore, electrochemical impedance spectroscopy (EIS) is an effective method for detecting physical, chemical, and biochemical changes in the environment [10]. This method is usable for manufacturing immunosensors [11], DNA sensors [12], glucose sensors [13], etc. When biomolecules (e.g., DNA, with an isoelectric point of 4.5) are deposited on $\mathrm{ZnO}$ nanostructures (isoelectric point of 9.5), interactions of the biomoleculenanostructure electronic subsystems occur, changing the local potential. The process of biochemical reactions on $\mathrm{ZnO}$ nanostructures also leads to changes in electrical parameters, reflected in the frequencydependent impedance of the system. Some authors argue that the change in resistance can be detected only at very small (about $20 \mathrm{~mm}$ ) distances above the electrode surfaces, requiring the spacing between the electrodes to be reduced to a few microns [14]. However, in other works, a significant change in impedance is observed on sensors where the interval between two adjacent electrodes is about a millimetre [15]. In our opinion, sensor sensitivity is largely determined by the morphology of nanostructures that interact with the analyte, the general geometry of the device, and the specific processes that take place on the electrode, as shown in our previous article [1]. Withal, changes in morphology can alter wettabilities of pore surfaces. This phenomenon could be important in electrochemical sensor and biosensor applications.

The main focus of this study is to examine the influence of $\mathrm{ZnO}$ nanostructure morphologies on the wettability and sensitivity of sensors based on EIS. By the term "sensitivity," we mean the value of the signal that is detected by the device resulting from processes occurring on the working surface. As a target substance, we choose distillate water, which does not chemically interact with $\mathrm{ZnO}$; although, it can form structured monolayers at the $\mathrm{ZnO}$-water interface [16], 
[17]. Thereby, water, wetting the nanostructured $\mathrm{ZnO}$ films, penetrates the gaps between the nanostructures. Penetration rate depends on a number of factors, including shape, size, and density of nanostructures and surface conditions of $\mathrm{ZnO}$, such as exposure to ultraviolet irradiation [18], [19]. Liquid penetration dynamics in the nanostructured film can be detected by EIS as the replacement of air in the voids by liquid must lead to a change in the impedance of the system. This phenomenon is useful in biosensors for wastewater monitoring. Furthermore, water can be used as a solvent for various biological solutions [20], [21]. In this case, biomolecules settle on the $\mathrm{ZnO}$ surface and change the wetting conditions of the nanostructures with water, altering the penetration dynamics of the liquid into nanostructure pores and, accordingly, changing the system impedance. Therefore, the concentration of biomolecules in the solution can be determined from the impedance change of the system. In addition, this method allows the saturation point (moment in time when the liquid completely fills air pockets of the nanostructured surface at the contact site) to be determined. In this paper, we describe the change in impedance of the system as a result of water penetration into depths of nanostructured films with different morphologies.

\section{EXPERIMENTAL}

\subsection{Materials}

Zinc nitrate hexahydrate $\left(\mathrm{Zn}\left(\mathrm{NO}_{3}\right)_{2} \cdot 6 \mathrm{H}_{2} \mathrm{O}\right.$; $\geq 99.0 \%$ CAS Number: 10196-18-6), zinc acetate $\left(\mathrm{Zn}\left(\mathrm{CH}_{3} \mathrm{COOH}\right)_{2} \cdot 2 \mathrm{H}_{2} \mathrm{O} ; \geq 99.0 \%\right.$, CAS: 5970-45-6), hexamethylenetetramine $\left(\mathrm{C}_{6} \mathrm{H}_{12} \mathrm{~N}_{4}\right.$; HMTA, $\geq 99.0 \%$, CAS Number: 100-97-0), and urea $\left(\mathrm{NH}_{2} \mathrm{CONH}_{2} \geq 99.0 \%\right.$, CAS Number: 57-13-6) were purchased

\subsection{Synthesis of ZnO Nanostructures}

In this research, six different morphologies of $\mathrm{ZnO}$ nanostructures such as nanoneedles, small diameter rods (thin rods), large diameter rods (thick rods), nanotubes, plates and a plain thin film were selected for the study of the wetting processes. Standard microscope slides $(76 \times 26 \mathrm{~mm})$ were used as samples substrates. First, $250 \mathrm{~nm}$ of chromium was deposited by DC magnetron sputtering through a patterned gate metal shadow mask onto pre-cleaned glass substrates by the LAB18 thin film deposition system (Kurt J. from Sigma Aldrich Co. All chemicals are analytical grade and used as received without further purification. Deionized and double-distilled water was self-made in the laboratory. Distilled water was purchased from Delfin Group.

Lesker, USA). As a result, planar concentric $\mathrm{Cr}$ electrodes of $500 \mu \mathrm{m}$ in width and 500 $\mu \mathrm{m}$ in spacing without galvanic contact were obtained (Fig. 1(6)). Secondly, the seed layer was prepared by coating $\mathrm{Cr}$ electrodes with zinc acetate solution in ethanol at $25 \mathrm{mmol}$ concentration through the patterned shadow mask (made of metallic and rubber liners (gaskets), then washed with pure ethanol and dried in a nitrogen stream. The procedure was repeated three times and then samples were calcinated at $200{ }^{\circ} \mathrm{C}$ for $30 \mathrm{~min}$ and 
slowly cooled to room temperature.

Following seeding onto metalic electrodes $\mathrm{ZnO}$ nanostructures were grown by a hydrothermal method [22]. Generally for $\mathrm{ZnO}$ nanostructure synthesis zinc nitrate hexahydrate and hexamethylenetetramine aqueous solution are commonly used. Here, $\mathrm{Zn}\left(\mathrm{NO}_{3}\right)_{2}$ serves as a source of $\mathrm{Zn}^{2+}$ ions, water - a source for $\mathrm{O}^{2-}$ ions, and HMTA acts as a slowly decomposing weak base, which maintains the weakly alkaline environment in the solution and provides the desired amount of $\mathrm{OH}^{-}$ions. A glass vessel with a lid was used as a chemical bath that was placed in a programmed Linn High Therm (Germany) oven preheated to $90{ }^{\circ} \mathrm{C}$. The $\mathrm{ZnO}$ seeded substrates were placed at an angle against the vessel, with the seed layer facing down. Depending upon the concentration of the reactants and the temperature of growth, the formation of $\mathrm{ZnO}$ nanostructures with different morphology was observed.

Thick rods: $\mathrm{ZnO}$ nanorod arrays were synthesized in $0.1 \mathrm{M}$ equimolar aqueous solution of zinc nitrate hexahydrate and HMTA at $90{ }^{\circ} \mathrm{C}$ for $3 \mathrm{~h}$.

Thin rods: For obtaining thin rods $0.05 \mathrm{M} \mathrm{Zn}\left(\mathrm{NO}_{3}\right)_{2}+0.2 \mathrm{M}$ HMTA solution were used. The growth process was carried out at $90^{\circ} \mathrm{C}$ for $3 \mathrm{~h}$.

Tubes: $\mathrm{ZnO}$ nanotubes were obtained by using a self-selective etching method with lowering temperatures of growth during the hydrothermal process. This method is based on $\mathrm{pH}$ level change after a decrease in growth temperature [23]-[27]. For the experiment $0.2 \mathrm{M}$ equimolar $\mathrm{Zn}\left(\mathrm{NO}_{3}\right)_{2}$ and HMTA concentration were used. At the first stage, in relatively short time (3h) at $90{ }^{\circ} \mathrm{C}$ temperature the growth process of $\mathrm{ZnO}$ nanorods intensively occurs in both lateral

\subsection{Characterization}

The surface morphology and structural properties were studied using a scanning and axial directions. At the second stage at $50{ }^{\circ} \mathrm{C}$ in a significantly longer time period, compared to the first stage $(18 \mathrm{~h})$, the aging process occurs: $\mathrm{ZnO}$ metastable planes are etched with residual chemicals of growth solution.

Needles: As indicated above, the main role of HMTA is to provide $\mathrm{OH}$ - ions; therefore, it was hypothesized that increasing the HMTA concentration in growth solution would cause the $\mathrm{pH}$ increase without the use of an additional chemical, like ammonia or hydroxides. $\mathrm{ZnO}$ nanoneedles arrays were synthesized in $0.05 \mathrm{M} \mathrm{Zn}\left(\mathrm{NO}_{3}\right)_{2}+0.2 \mathrm{M}$ HMTA solution at $90{ }^{\circ} \mathrm{C}$ for $3 \mathrm{~h}$.

Plates: This morphology was obtained by replacing HMTA with urea, which promoted lateral growth and inhibited growth in the axial (c-axis) direction during the hydrothermal synthesis process. As a result, the total surface area of (0002) planes increased, but the lateral surface area decreased. 2D plate-like $\mathrm{ZnO}$ nanostructures were grown in aqueous solution of $30 \mathrm{mM}$ zinc nitrate and $0.5 \mathrm{M}$ urea at $90^{\circ} \mathrm{C}$ for $3 \mathrm{~h}$. After hydrothermal growth samples were annealed at $250^{\circ} \mathrm{C}$ for $2 \mathrm{~h}$, in order to calcine the obtained zinc carbonate to $\mathrm{ZnO}$.

After hydrothermal growth, all obtained samples were thoroughly washed with distilled water in order to remove traces of the growth solution from the surface of nanostructures. Then, the samples were dried in oven for one hour at a temperature of $90^{\circ} \mathrm{C}$ in order to get rid of residual water accumulated on their surface and in the space between the nanostructures.

The unstructured $150 \mathrm{~nm}$ thick ZnO film was obtained by magnetron sputtering (MS) mode in Kurt J. Lesker LAB 18 sputtering system.

electron microscopy (SEM, TESCANVEGA LMU II operated at $30 \mathrm{kV}$ ). 
Surface wettability of obtained $\mathrm{ZnO}$ thin films was identified by measuring the water contact angle (WCA) at ambient temperature using the sessile drop method by an optical microscope (Motic BA50-X). A $3 \mu \mathrm{L}$ droplet of distilled water was placed on the sample surface, and digital images of the droplet silhouette were captured with the implemented camera every minute for 10 minutes. The WCA was determined using the Motic Images Plus image processing software. An average of five measurements, performed at different spots on the same sample, was adopted as the WCA. Surface roughness and surface coverage of nanostructures were analysed with ImageJ Analysis software.

Additional experiments were carried out to determine the impact of surface mor- phology on the wetting dynamics by EIS. Electrochemical measurements were performed at room temperature with a VersaSTAT 3 (Princeton Applied Research) potentiostat/galvanostat. Structures of the electrical measurement cell and electrodes are shown in Fig. 1. ZnO-coated $\mathrm{Cr}$ electrodes were used as the counter and working electrodes. A drop of the target liquid $(250 \mu \mathrm{l})$ was pelleted on the cell with the electrodes, and the dependence of the phase shift on frequency was measured immediately and repeated every minute for $10 \mathrm{~min}$ utes. An average of eight independent measurements, performed at different spots on the same sample, was adopted to establish dependency between wetting dynamics and surface structure of all $\mathrm{ZnO}$ nanostructure morphologies.

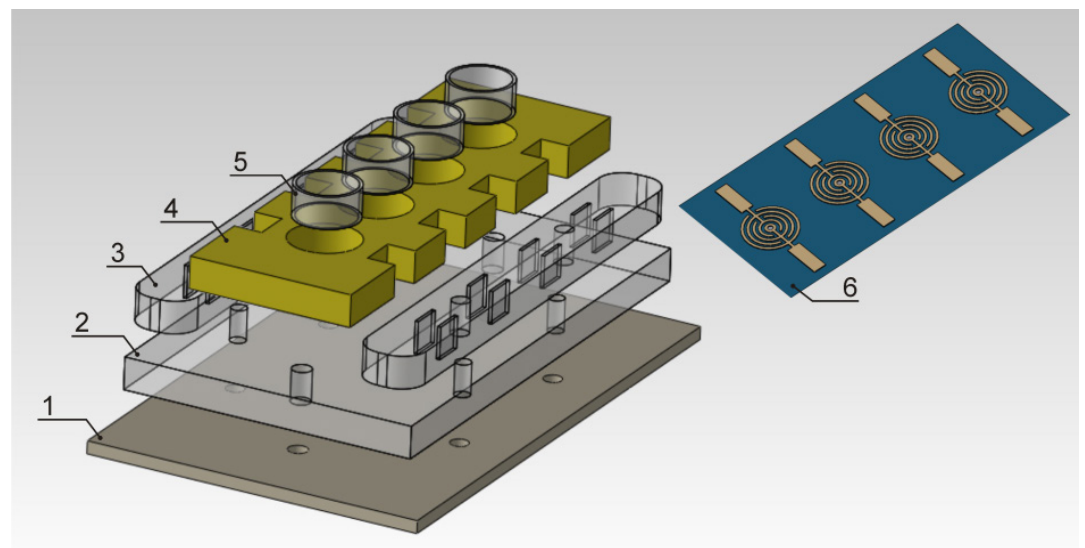

Fig. 1. Structure of the electrical measurement cell (left) and electrodes (right). The measurement cell consists of the following parts: a corps (1), an interlayer (2) with push-in contacts (3), a sealing rubber mask (4) with replaceable plastic cylinders (5). Sample (6) consists of four electrodes, which allow four independent analyte measurements to be performed consistently [1].

\section{RESULTS AND DISCUSSION}

\subsection{Wettability versus Surface Morphology}

Surface morphologies of the prepared samples were characterised using SEM. Figure 2 demonstrates six different types of
$\mathrm{ZnO}$ nanostructures: nanoneedles (Fig. 2a), small diameter rods (Fig. 2b), large diameter rods (Fig. 2c), nanotubes (Fig. 2d), plates 
(Fig. 2e), and a thin $150 \mathrm{~nm}$ MS layer (Fig. 2f). As seen in Fig. 2, the nanoneedle array was not aligned. The average diameter of the base stems and their heads were around 350 and $60 \mathrm{~nm}$, respectively. The average length of the $\mathrm{ZnO}$ nanoneedle stems was around 1 $\mu \mathrm{m}$. $\mathrm{ZnO}$ nanorod arrays and nanotubes with hexagonal structures were preferentially oriented towards the c-axis perpendicular to the glass substrate. The smaller nanorods had an average diameter of $\sim 100 \mathrm{~nm}$, while larger nanorods and nanotubes were around $1 \mu \mathrm{m}$. The $\mathrm{ZnO}$ nanoplates were very dense, with thicknesses of approximately $300 \mathrm{~nm}$. The nanoscale roughness on the film surfaces reduced the contact area between the solid and droplet; thereby, a large amount of air was entrapped into the space below the droplet, effecting the wettability of the surface.
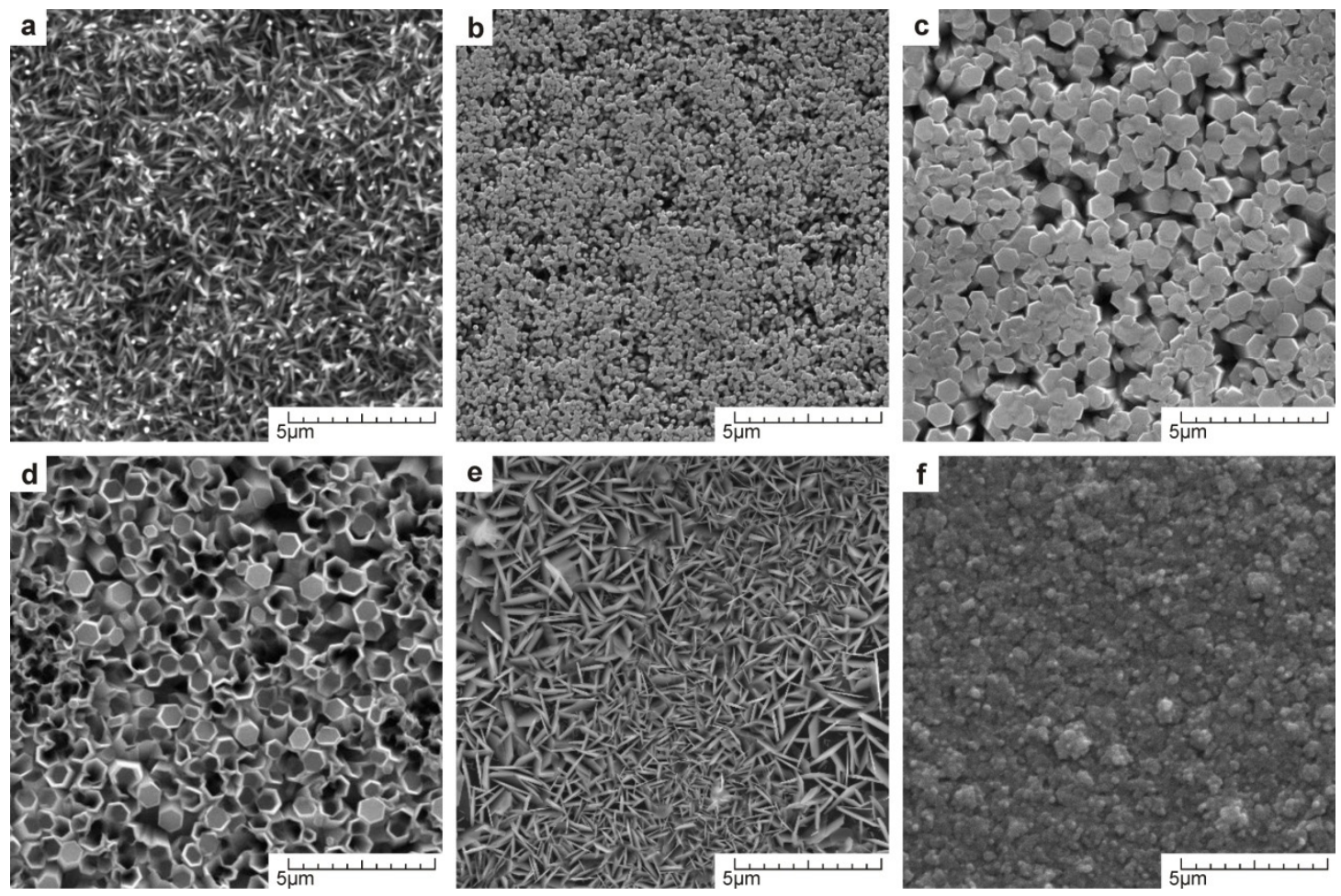

Fig. 2. Morphology of $\mathrm{ZnO}$ thin films: (a) nanoneedles, (b) thin rods, (c) thick rods, (d) nanotubes, (e) nanoplates, and (f) the MS thin film.

Myo Tay Zar Myint et al. point out that for homogeneous, unstructured, and untreated $\mathrm{ZnO}$ thin films, consisting of many chaotically oriented nanocrystallites, the intrinsic WCA is $26 \pm 3^{\circ}$ [28]-[31]. Some other articles refer to different WCA values, which are, in general, smaller than $50^{\circ}$, indicating the hydrophilic nature of the $\mathrm{ZnO}$ surface [32]. However, in the case of nanostructured $\mathrm{ZnO}$ thin films, the surface can exhibit both hydrophobic and hydrophilic behaviour depending on the nanostructure parameters, and the transition from hydrophilic to hydrophobic conditions can be realised by micro- and nanostructuring of the surface [33]-[38].

In our opinion, there are two main factors that determine the wettability of an individual sample: the surface coverage of the nanostructures (percentage ratio of the 
solid phase and voids) and the nanostructure morphologies, which determine the number of active sites capable of binding hydroxyl groups. The first hypothesis has also been proven in previous work [28], which indicates the possibility of changing the wettability of the $\mathrm{ZnO}$ surface by micro/ nanostructuring [39]. As mentioned in the article, surfaces with nanostructure cover- age areas less than $39 \%$ show hydrophobic character, and the classical Cassie-Baxter criteria can be applied to the so-called "Fakir" surface (Fig. 3a) [28], [40]. However, for high solid area fractions $(>40 \%)$, the surface was completely wet, and water penetrated into the pores. In such a case, Wenzel gave a wettability description of the films (Fig. 3b).
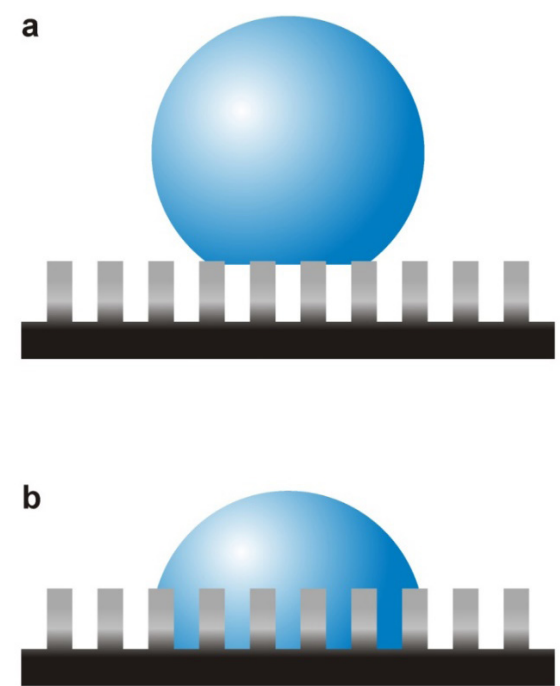

Fig. 3. Schematic illustration of (a) Cassie-Baxter and (b) Wenzel regimes.

These hypotheses have also been confirmed in our experiment, where samples are represented by several rod-type nanostructures that differ in size and, respectively, in the degree of surface coverage (Figs. $2 \mathrm{a}-\mathrm{d}$ ). Figure 4 shows the dependence of the wetting angle on the time for each morphology. The highest WCA value was observed for $\mathrm{ZnO}$ nanoneedle (Fig. 2a) arrays: $127^{\circ}$ at the beginning of the measurement and $70^{\circ}$ after 10 minutes (Fig. 4, red curve). The surface structure of this sample can be characterised by the lowest degree of surface coverage $(23 \%)$. The hydrophobic effect was also enhanced by the existence of the 'fakir' or 'lotus' surfaces, when the interaction between the droplets and solid surface was reduced to the point contact because of the needle-like nanostructure shapes. In the case of thin rod shaped nanostructures (Fig. 2b), an increase in surface coverage to $38 \%$ was observed, promoting a decrease in a wetting angle and total omission of the graph for angle-time dependence indicating a decrease in hydrophobic properties compared to nanoneedles. The value of the wetting angle changed from $86^{\circ}$ to $57^{\circ}$ within 10 minutes (Fig. 4, blue curve). The decrease in the initial WCA value compared to nanoneedles may be explained by the increase in contact surface due to the flat top of the nanorods, leading to a stronger interaction between the water and solid surface. In the case of thick rods (Fig. 2c), the surface coverage increased up to $87 \%$. Therefore, in contrast with previous rod-shaped morphologies, hydrophilic surface behaviour was observable; the wet- 
ting angle did not exceed $19^{\circ}$ at the starting point or $7.5^{\circ}$ after 10 minutes (Fig. 4, green curve). In the case of the MS thin film (Fig. $2 \mathrm{f}$ ), the WCA was $44^{\circ}$ at the starting point and remained almost constant over time (Fig. 4, black line). Minor changes may have been caused by slight spreading of the droplet on the surface.

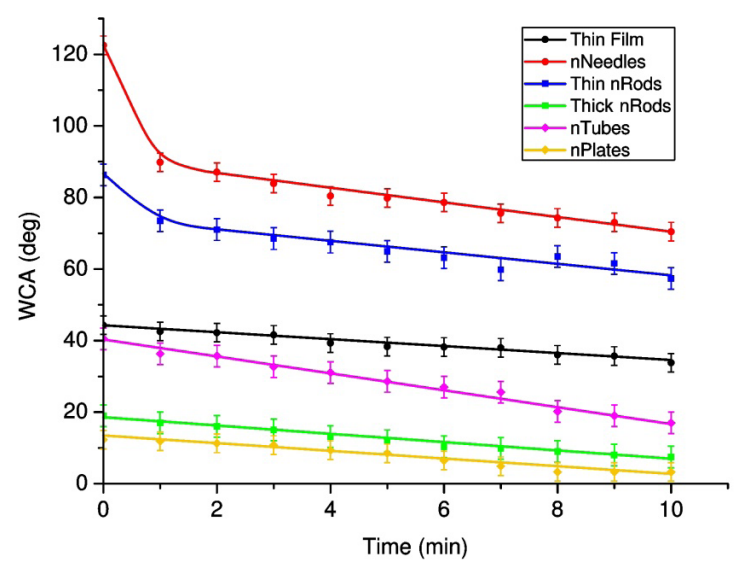

Fig. 4. WCA changes over time for different $\mathrm{ZnO}$ nanostructure morphologies.

Comparing large diameter nanorod and MS thin film wetting angle values, a difference of $30^{\circ}$ was observed for the starting point of the wetting angle with only a very small surface coverage difference between the two samples $(\approx 10 \%)$. This difference indicates a stronger interaction between the liquid and solid surface for the nanostructured sample. Importantly, the surface coverage is influential but is not the only factor that determines surface wetting; nanostructure morphology also accounts for significant contributions. As it is known, $\mathrm{ZnO}$ nanostructures have the form of a hexagonal prism, in which the two most significant plane groups can be distinguished. The first group includes nonpolar side $\{1010\}$ planes, containing both zinc ions and oxygen and being electrically neutral and chemically stable. The second group includes metastable polar $\{0001\}$ planes, consisting of either oxygen ions or zinc ions (Zn-terminated or O-terminated planes), which have an electric charge and are chemically more active. The structure of the MS thin film can be described as a set of randomly oriented crystallites. Meanwhile, the nanorod array (nanostructured sample) is compositionally arranged and, in a horizontal plane, is represented by a metastable plane [0002], containing a large number of active adsorption bonds on its surface. An important role of this plane was also confirmed by WCA measurements of $\mathrm{ZnO}$ nanoplate arrays, for which, considering the preferential growth direction, this plane was dominant. This morphology showed the hydrophilic behaviour and even smaller contact angle values than in the previous case: $12^{\circ}$ at the beginning of the experiment and $3^{\circ}$ after 10 minutes (Fig. 4, yellow curve). This indicates that both coverage and nanostructure morphology play an important role.

In particular, the $\mathrm{ZnO}$ nanotube morphology (Fig. 2d) deserves special attention. Despite the low percentage of surface coverage by these nanostructures $(40 \%)$, as well as a decreased total area of the [0002] plane, this morphology revealed strongly hydrophilic behaviour: $40^{\circ}$ at the beginning of process and $17^{\circ}$ after 10 minutes (Fig. 4 , pink curve). This phenomenon could be explained by the presence of a large num- 
ber of active adsorption centres that are related to structure defects formed on the inner walls of nanotubes during the etching process. Such centres have higher adsorp- tion energy values compared to non-polar centres and provide stronger interaction between water and solid.

\subsection{Analysis of the Wetting Process by EIS}

The aim of this study is to describe the usage of EIS as an alternative method for analysing the wetting processes of nanostructured surfaces. This technique makes it possible to describe, with great accuracy, the wetting processes, not only on the surface, but also in the volume of the nanostructured layer due to changes in the sample resistance in the process of filling the pores with liquid. Furthermore, this method allows us to track dynamics of the
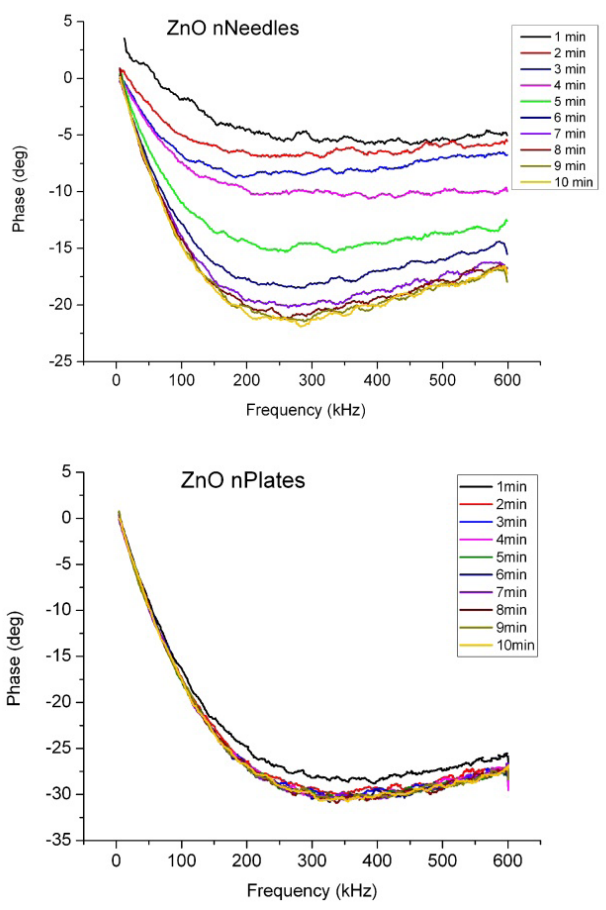

wetting process for each morphology, as well as determine the saturation time, i.e., the moment in time where complete wetting of all structures occurs. Also, this method allows us to describe the dynamics of intermediate processes that occur during wetting and the transition from the Cassie-Baxter model at the beginning of the process to the Wenzel model at the end, when all nanostructure voids are completely filled with water.
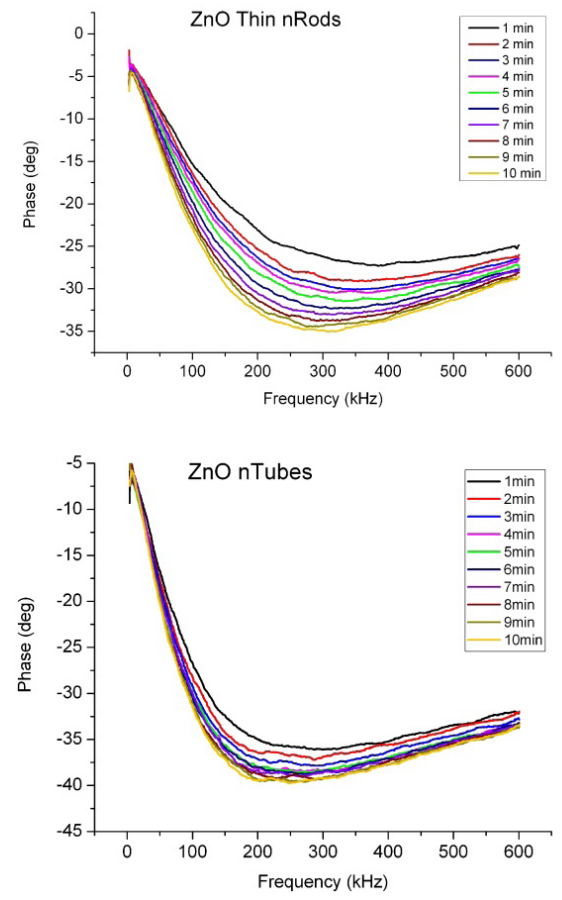

Fig. 5. EIS spectra for the wetting process for different $\mathrm{ZnO}$ nanostructure morphologies.

Figure 5 shows the phase dependence curves over time for two hydrophobic (upper row) and two hydrophilic (bottom row) $\mathrm{ZnO}$ nanostructure morphologies. As can be seen, the wetting process is uneven and non-linear. In the first few minutes, there is a large difference between the phases of adjacent curves. However, over time, this step decreases until the phase difference becomes minimal, and the curves coincide. This moment can be defined as the moment of saturation, which character- 
ises complete wetting of the nanostructured sample. As expected, in the case of hydrophobic surfaces, the dynamics of phase change is more readily expressed, and the saturation state is characterised by a longer time interval (7-9 minutes). In the case of hydrophilic surfaces, the saturation occurs much faster (2-4 minutes), and the phase value changes are smaller.

To better understand the degree of hydrophobicity of the structures, the graph of the relative change in impedance versus time is very descriptive (Fig. 6). This curve characterises the change in impedance of the full system relative to the zero point, which corresponds to the beginning of the measurements and characterises the amount of liquid absorbed by the entire volume of the nanostructured sample. In the case of the plain film, the relative changes in impedance are minimal and occur only due to absorption of liquid by small irregularities arising from the polycrystalline structure of the sample. For large diameter rods, the relative changes in impedance are caused by the penetration of water into the voids between nanostructures. However, as the oriented nanorods are dense and the voids are narrow, the dynamics is poorly expressed. In the case of nano- plates, there is a slight increase in relative impedance changes over time. The relative changes in impedance do not exceed $12 \%$ and the rapid wetting is caused by both a decrease of surface coverage (increase in porosity) and an increase in the total area of the metastable [002] plane. At the beginning of the measurement, voids are almost completely filled with water, so in case of hydrophilic nanostructures further impedance values practically do not change over time. For nanotubes, the wetting process is significantly slower than for nanoplates and thick rods and is mainly provided by cavities in the nanostructures reducing the degree of surface filling compared with the same diameter nanorods. However, the morphology still shows hydrophilic properties due to the presence of a large number of structural defects formed on the inner walls during etching process which prevent the manifestation of completely hydrophobic properties. This fact shows good conformity with theory and WCA measurements described in Section 3.1. For small diameter nanorods and nanoneedles, the relative increase in impedance is caused by a decrease in the surface coverage, correlating well with WCA measurements (Fig. 4).

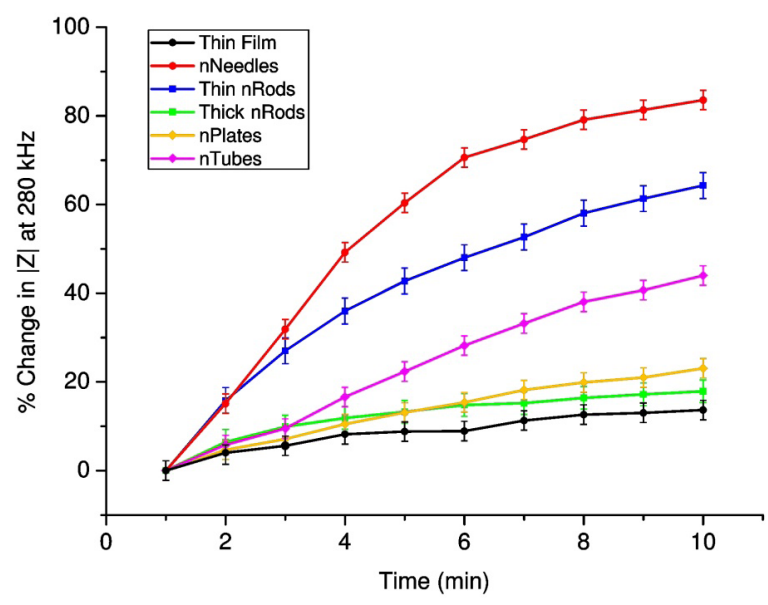

Fig. 6. Relative change (in \%) of the impendance module $|\mathrm{Z}|$ versus time for different $\mathrm{ZnO}$ nanostructure morphologies (as indicated in the legend) at $280 \mathrm{kHz}$. 
The surface of nanostructured $\mathrm{ZnO}$ thin films exhibits both hydrophilic and hydrophobic wetting behaviour, depending on nanostructure form, size, and orientation. $\mathrm{ZnO}$ nanoneedles with WCAs of $127^{\circ}$ at the beginning of the experiment, as well as thin nanorods with WCAs of $86^{\circ}$, are hydrophobic. In contrast, $\mathrm{ZnO}$ thin film $\left(44^{\circ}\right)$, thick nanorods $\left(19^{\circ}\right)$, nanoplates $\left(12^{\circ}\right)$, and nanotubes $\left(40^{\circ}\right)$ exhibit hydrophilic behaviour.

We have demonstrated the possibility of investigate wetting processes by EIS. With great accuracy, this technique describes both surface wetting and processes occurring at the volume of the nanostructured layer. Thus, this method allows us to describe dynamics of the wetting process when the Cassie-Baxter wetting model at the beginning of the process is gradually replaced with a Vensel model at the end of the process, thus facilitating the determination of the saturation value (moment in time when liquid completely fills air pockets of the nanostructured surface). In cases of hydrophobic behaviour, saturation occurs within 7-9 minutes, but hydrophilic morphologies are fully wetted within 2-4 minutes.

The results obtained by EIS correspond to the results obtained by the optical method.

\section{ACKNOWLEDGEMENTS}

This study has been supported by internal research grant No. 14-95/2021/10 of Daugavpils University "Development of the Nanostructured Metal Oxide Coatings and Their Application in Optical Sensing for Heavy Metal Detection".

\section{REFERENCES}

1. Krasovska, M., Gerbreders, V., Mihailova, I., Ogurcovs, A., Sledevskis, E., Gerbreders, A., \& Sarajevs, P. (2018). ZnO-NanostructureBased Electrochemical Sensor: Effect of Nanostructure Morphology on the Sensing of Heavy Metal Ions. Beilstein Journal of Nanotechnology, 9, 2421-2431. DOI:10.3762/bjnano.9.227.

2. Tereshchenko, A., Bechelany, M., Viter, R., Khranovskyy, V., Smyntyna, V., Starodub, N., \& Yakimova, R. (2016). Optical Biosensors Based on $\mathrm{ZnO}$ Nanostructures: Advantages and Perspectives. A Review. Sensors and Actuators B: Chemical, 229, 664-677. DOI:10.1016/j.snb.2016.01.099.

3. Zaidi, S. A., \& Shin, J. H. (2016). Recent
Developments in Nanostructure Based Electrochemical Glucose Sensors. Talanta, 149, 30-42. DOI:10.1016/j. talanta.2015.11.033.

4. Arya, S. K., Saha, S., Ramirez-Vick, J. E., Gupta, V., Bhansali, S., \& Singh, S. P. (2012). Recent Advances in $\mathrm{ZnO}$ Nanostructures and Thin Films for Biosensor Applications: Review. Analytica Chimica Acta, 737, 1-21. DOI:10.1016/j.aca.2012.05.048.

5. Mihailova, I., Gerbreders, V., Tamanis, E., Sledevskis, E., Viter, R., \& Sarajevs, P. (2013). Synthesis of $\mathrm{ZnO}$ Nanoneedles by Thermal Oxidation ofZn Thin Films. Journal of Non-Crystalline Solids, 377, 212-216. DOI:10.1016/j.jnoncrysol.2013.05.003. 
6. Mihailova, I., Gerbreders, V., Bulanovs, A., Tamanis, E., Sledevskis, E., Ogurcovs, A., \& Sarajevs, P. (2014). Controlled growth of well-aligned $\mathrm{ZnO}$ nanorod arrays by hydrothermal method. In: Eighth International Conference on Advanced Optical Materials and Devices, (pp. 1-8), 25-27 August 2014, 9421, 94210A. Riga, Latvia: SPIE. DOI:10.1117/12.2083960.

7. Alvi, N. H., Hassan, W. ul, Farooq, B., Nur, O., \& Willander, M. (2013). Influence of Different Growth Environments on the Luminescence Properties of $\mathrm{ZnO}$ Nanorods Grown by the Vapor-Liquid-Solid (VLS) Method. Materials Letters, 106, 158-16. DOI:10.1016/j.matlet.2013.04.074.

8. Abbas, K. N., Bidin, N., Sabry, R. S., AlAsedy, H. J., Al-Azawi, M. A., \& Islam, S. (2016). Structures and Emission Features of High-Density ZnO Micro/Nanostructure Grown by an Easy Hydrothermal Method. Materials Chemistry and Physics, 182, 298-307.DOI:10.1016/j. matchemphys.2016.07.035.

9. Baruah, S., \& Dutta, J. (2009). Hydrothermal Growth of $\mathrm{ZnO}$ Nanostructures. Science and Technology of Advanced Materials, 10 (1), 013001. DOI:10.1088/14686996/10/1/013001.

10. Katz, E., \& Willner, I. (2003). Probing Biomolecular Interactions at Conductive and Semiconductive Surfaces by Impedance Spectroscopy: Routes to Impedimetric Immunosensors, DNA-Sensors, and Enzyme Biosensors. Electroanalysis, 15 (11), 913947. DOI:10.1002/elan.200390114.

11. Lin, D., Tang, T., Jed Harrison, D., Lee, W. E., \& Jemere, A. B. (2015). A Regenerating Ultrasensitive Electrochemical Impedance Immunosensor for the Detection of Adenovirus. Biosensors and Bioelectronics, 68, 129-134. DOI:10.1016/j. bios.2014.12.032.

12. Kafka, J., Pänke, O., Abendroth, B., \& Lisdat, F. (2008). A Label-Free DNA Sensor Based on Impedance Spectroscopy. Electrochimica Acta, 53 (25), 7467-7474. DOI:10.1016/j.electacta.2008.01.031.

13. Ni, Y., Xu, J., Liang, Q., \& Shao, S. (2017). Enzyme-Free Glucose Sensor Based on
Heteroatom-Enriched Activated Carbon (HAC) Decorated with Hedgehog-Like NiO Nanostructures. Sensors and Actuators B: Chemical, 250, 491-498. DOI:10.1016/j. snb.2017.05.004.

14. Sanguino, P., Monteiro, T., Bhattacharyya, S. R., Dias, C. J., Igreja, R., \& Franco, R. (2014). $\mathrm{ZnO}$ Nanorods as Immobilization Layers for Interdigitated Capacitive Immunosensors. Sensors and Actuators B: Chemical, 204, 211-217. doi:10.1016/j.snb.2014.06.141.

15. Jacobs, M., Muthukumar, S., Munje, R., Quadri, B., \& Prasad, S. (2014). Analysis of nanotextured $\mathrm{ZnO}$ surfaces for biosensing applications. In: 14th IEEE International Conference on Nanotechnology, (pp. 515520), 18-21 August 2014. Toronto, Canada: IEEE. DOI:10.1109/nano.2014.6968149.

16. Raymand, D., van Duin, A. C. T., Spångberg, D., Goddard, W. A., \& Hermansson, K. (2010). Water Adsorption on Stepped $\mathrm{ZnO}$ Surfaces from MD Simulation. Surface Science, 604 (9-10), 741-752. DOI:10.1016/j.susc.2009.12.012.

17. Hamid, S. B. A., Teh, S. J., \& Lai, C. W. (2017). Photocatalytic Water Oxidation on ZnO: A Review. Catalysts, 7 (12), 93. DOI:10.3390/catal7030093.

18. Bhavsar, K., Ross, D., Prabhu, R., \& Pollard, P. (2015). LED-Controlled Tuning of $\mathrm{ZnO}$ Nanowires' Wettability for Biosensing Applications. Nano Reviews, 6 (1), 26711. DOI:10.3402/nano.v6.26711.

19. Khranovskyy, V., Ekblad, T., Yakimova, R., \& Hultman, L. (2012). Surface Morphology Effects on the Light-Controlled Wettability of $\mathrm{ZnO}$ Nanostructures. Applied Surface Science, 258 (20), 8146-8152. DOI:10.1016/j.apsusc.2012.05.011.

20. Ejeian, F., Etedali, P., Mansouri-Tehrani, H.A., Soozanipour, A., Low, Z.-X., Asadnia, M., .... \& Razmjou, A. (2018). Biosensors for Wastewater Monitoring: A Review. Biosensors and Bioelectronics, 118, 66-79. DOI:10.1016/j.bios.2018.07.019.

21. Duta, L., Popescu, A. C., Zgura, I., Preda, N., \& Mihailescu, I. N. (2015). Wettability of Nanostructured Surfaces. Wetting and Wettability, Intech Open, 207-252. DOI:10.5772/60808. 
22. Krasovska, M., Gerbreders, V., Sledevskis, E., Gerbreders, A., Mihailova, I., Tamanis, E., \& Ogurcovs, A. (2020). Hydrothermal Synthesis of $\mathrm{ZnO}$ Nanostructures with Controllable Morphology Change. CrystEngComm., 28 (8), 1346-1358. DOI:10.1039/c9ce01556f.

23. Krasovska, M., Gerbreders, V., Paskevics, V., Ogurcovs, A., \& Mihailova, I. (2015). Obtaining a Well-Aligned $\mathrm{ZnO}$ Nanotube Array Using the Hydrothermal Growth Method. Latvian Journal of Physics and Technical Sciences, 52 (5), 28-40. DOI:10.1515/lpts-2015-0026.

24. Gerbreders, V., Krasovska, M., Mihailova, I., Ogurcovs, A., Sledevskis, E., Gerbreders, A., ... \& Plaksenkova, I. (2019). ZnO Nanostructure-Based Electrochemical Biosensor for Trichinella DNA Detection. Sensing and Bio-Sensing Research, 100276. DOI:10.1016/j.sbsr.2019.100276.

25. Chae, K.-W., Zhang, Q., Kim, J. S., Jeong, Y.-H., \& Cao, G. (2010). Low-Temperature Solution Growth of $\mathrm{ZnO}$ Nanotube Arrays. Beilstein Journal of Nanotechnology, 1, 128-134. DOI:10.3762/bjnano.1.15.

26. Roza, L., Rahman, M. Y. A., Umar, A. A., \& Salleh, M. M. (2015). Direct Growth of Oriented $\mathrm{ZnO}$ Nanotubes by Self-Selective Etching at Lower Temperature for PhotoElectrochemical (PEC) Solar Cell Application. Journal of Alloys and Compounds, 618, 153158. DOI:10.1016/j.jallcom.2014.08.113.

27. Wang, H., Li, G., Jia, L., Wang, G., \& Tang, C. (2008). Controllable Preferential-Etching Synthesis and Photocatalytic Activity of Porous $\mathrm{ZnO}$ Nanotubes. The Journal of Physical Chemistry C, 112(31), 1173811743. DOI:10.1021/jp803059k.

28. Myint, M. T. Z., Kumar, N. S., Hornyak, G. L., \& Dutta, J. (2013). Hydrophobic/ Hydrophilic Switching on Zinc Oxide Micro-Textured Surface. Applied Surface Science, 264, 344-348. DOI:10.1016/j. apsusc.2012.10.024.

29. Patel, K. H., \& Rawal, S. K. (2016). Exploration of Wettability and Optical Aspects of ZnO Nano Thin Films Synthesized by Radio Frequency Magnetron Sputtering. Nanomaterials and Nanotechnology, 6, 22. DOI:10.5772/62804.
30. Han, J., \& Gao, W. (2008). Surface Wettability of Nanostructured Zinc Oxide Films. Journal of Electronic Materials, 38 (4), 601-608. DOI:10.1007/s11664-0080615-0.

31. Shaban, M., Zayed, M., \& Hamdy, H. (2017). Nanostructured $\mathrm{ZnO}$ Thin Films for Self-Cleaning Applications. RSC Advances, 7 (2), 617-631. DOI:10.1039/c6ra24788a.

32. Lin, L.-Y., Kim, H.-J., \& Kim, D.-E. (2008). Wetting Characteristics of ZnO Smooth Film and Nanowire Structure with and without OTS Coating. Applied Surface Science, 254 (22), 7370-7376. DOI:10.1016/j. apsusc.2008.05.337.

33. Subedi, D. P., Madhup, D. K., Sharma, A., Joshi, U. M., \& Huczko, A. (2012). Retracted: Study of the Wettability of $\mathrm{ZnO}$ Nanofilms. International Nano Letters, 2 (1), 117-122. DOI:10.1186/2228-5326-2-1.

34. Mao-Gang, G., Xiao-Liang, X., Zhou, Y., Yan-Song, L., \& Ling, L. (2010). Superhydrophobic Surfaces via Controlling the Morphology of $\mathrm{ZnO}$ Micro/Nano Complex Structure. Chinese Physics B, 19 (5), 056701. DOI:10.1088/16741056/19/5/056701.

35. Yang, P., Wang, K., Liang, Z., Mai, W., Wang, C., Xie, W., ... \& Song, J. (2012). Enhanced Wettability Performance of Ultrathin $\mathrm{ZnO}$ Nanotubes by Coupling Morphology and Size Effects. Nanoscale, 4 (18), 5755. DOI:10.1039/c2nr31380d.

36. Suresh Kumar, P., Sundaramurthy, J., Mangalaraj, D., Nataraj, D., Rajarathnam, D., \& Srinivasan, M. P. (2011). Enhanced Super-Hydrophobic and Switching Behavior of $\mathrm{ZnO}$ Nanostructured Surfaces Prepared by Simple Solution - Immersion Successive Ionic Layer Adsorption and Reaction Process. Journal of Colloid and Interface Science, 363 (1), 51-58. DOI:10.1016/j. jcis.2011.07.015.

37. Piech, M., Sounart, T. L., \& Liu, J. (2008). Influence of Surface Morphology on the Wettability of Microstructured ZnO-Based Surfaces. The Journal of Physical Chemistry C, 112 (51), 20398-20405. DOI:10.1021/ jp804815x. 
38. Zhou, X., Guo, X., Ding, W., \& Chen, Y. (2008). Superhydrophobic or Superhydrophilic Surfaces Regulated by Micro-Nano Structured $\mathrm{ZnO}$ Powders. Applied Surface Science, 255 (5), 33713374. DOI:10.1016/j.apsusc.2008.09.080.

39. Ennaceri, H., Wang, L., Erfurt, D., Riedel, W., Mangalgiri, G., Khaldoun, A., ... \& Ennaoui, A. (2016). Water-Resistant Surfaces Using Zinc Oxide Structured Nanorod Arrays with Switchable Wetting Property. Surface and Coatings Technology, 299, 169-176. DOI:10.1016/j.surfcoat.2016.04.056.
40. Singh, A., \& Singh, S. (2018). ZnO Nanowire-Coated Hydrophobic Surfaces for Various Biomedical Applications. Bulletin of Materials Science, 41(4). DOI:10.1007/ s12034-018-1611-5. 